\title{
Hemangiopericitoma de órbita
}

\author{
Orbitalhemangiopericytoma
}

\author{
Ana Paula Ximenes Alves ${ }^{1}$ \\ Paulo Roberto Félix ${ }^{2}$ \\ Antonio Augusto Velasco e Cruz
}

Faculdade de Medicina de Ribeirão Preto da Universidade de São Paulo.

${ }^{1}$ Doutora em Oftalmologia pelo Departamento de Oftalmologia e Otorrinolaringologia.

Médico contratado do Departamento de Patologia.

${ }^{3}$ Professor Associado, responsável pelo setor de Órbita e Oculoplástica do Departamento de Oftalmologia e Otorrinolaringologia.

Endereço para correspondência: Antonio Augusto V. Cruz - Departamento de Oftalmologia e Otorrinolaringologia, Hospital das Clínicas-Campus, $12^{\circ}$ andar Faculdade de Medicina de Ribeirão Preto da Universidade de São Paulo - Av. Bandeirantes 3900 - Ribeirão Preto (SP) - CEP 14049-900

\section{RESUMO}

Objetivo: Descrever um raro caso de hemangiopericitoma orbital. Métodos: Relato de caso de associação entre hemangipericitoma orbital e blefaroptose. Resultados: A exérese da neoplasia normalizou o posicionamento palpebral. Conclusões: Lesões orbitais anteriores são causas de blefaroptose por compressão do músculo elevador palpebral.

Descritores: Hemangiopericitoma; Neoplasias orbitárias/etiologia; Blefaroptose/complicações

\section{INTRODUÇ̃̃̃O}

Os hemangiopericitomas foram descritos pela primeira vez, em 1942, por Stout e Murray ${ }^{(1)}$. São tumores de ocorrência rara, que se originam a partir da proliferação de pericitos, ou seja, células que envolvem os capilares, dando-lhes suporte e regulando o seu lúmen.

Os hemangiopericitomas comumente aparecem nas extremidades inferiores, principalmente coxa. Além dessa localização, eles são comuns na fossa pélvica e no retroperitôneo ${ }^{(2)}$. A órbita é um local de rara ocorrência. Alguns autores o identificaram em 1\% das biópsias orbitárias, enquanto outros afirmam que estas lesões constituem cerca de $2 \%$ dos tumores orbitários $^{(2)}$.

Apesar de poderem acometer qualquer faixa etária, o aparecimento dos sintomas geralmente se dá entre a terceira e sexta décadas da vida, sob a forma de proptose lentamente progressiva e unilateral. Entre os sintomas associados, descreve-se, mais comumente, a ocorrência de dor leve e redução da acuidade visual $^{(2)}$.

Os exames de imagem não são patognomônicos. O padrão mais encontrado é o de uma lesão bem delimitada, que pode realçar com o uso de contrastes. Pode ser encontrada em qualquer lugar da órbita, mas tem predileção pela órbita superior, deslocando o olho para baixo. A histologia é fundamental para o diagnóstico ${ }^{(3)}$. Em relação à evolução, os hemangiopericitomas podem apresentar comportamento benigno ou maligno. No último caso, podem gerar metástases para órgãos distantes, como pulmão, osso e fígado, e levar a óbito ${ }^{(4)}$.

O objetivo do presente trabalho é descrever um caso de hemangiopericitoma orbitário e realçar as características clínicas e histopatológicas dessa neoplasia.

\section{RELATO DE CASO}

A paciente GTF, de 69 anos, procurou o serviço de órbita do Hospital das Clínicas da Faculade de Medicina de Ribeirão Preto da Universidade de 
São Paulo (HCFMRP-USP), em março de 1998, referindo inchaço progressivo na pálpebra superior esquerda (PSE), associado a dor no olho ipsilateral, há cerca de três anos. Negava doenças oculares anteriores. Estava em seguimento ambulatorial por ser portadora de doença diverticular do cólon, além de prolapso retal e uterino.

Ao exame, apresentava acuidade visual corrigida de 0,5 à direita e 0,8 à esquerda. Notava-se ptose palpebral à esquerda, associada à tumefação abaixo da porção central do rebordo orbitário esquerdo.

A distância entre o centro pupilar e a margem palpebral direita era de $3,0 \mathrm{~mm}$ à direita e de $1,0 \mathrm{~mm}$ à esquerda. $\mathrm{O}$ olho esquerdo estava nitidamente deslocado para baixo. Embora as versões estivessem normais e não houvesse diplopia, a paciente queixava-se de dor à movimentação do olho esquerdo. À biomicroscopia, observava-se opacificação subcapsular posterior à direita e à fundoscopia, notavamse drusas maculares e atrofia do epitélio pigmentar peripapilar bilateralmente.

A tomografia de órbita revelou estrutura ovalada, de contornos regulares, medindo $2,0 \mathrm{~cm} \mathrm{x} \mathrm{1,2} \mathrm{cm,} \mathrm{na} \mathrm{órbita} \mathrm{anterior} \mathrm{e}$ superior provocando afinamento do teto orbitário. Após a administração de contraste, notou-se reforço homogêneo da lesão (Figura 1).

Foi realizada exérese total da lesão, através de incisão no sulco palpebral superior e dissecção extra-periostal. A lesão mostrava-se bem delimitada e de coloração vinhosa. Não houve sangramento importante durante o procedimento.

O exame anátomo-patológico da massa evidenciou proliferação de células fusiformes agrupadas em feixes e intercaladas por múltiplos e pequenos canais vasculares, revestidos por células endoteliais aplanadas. A lesão apresentava

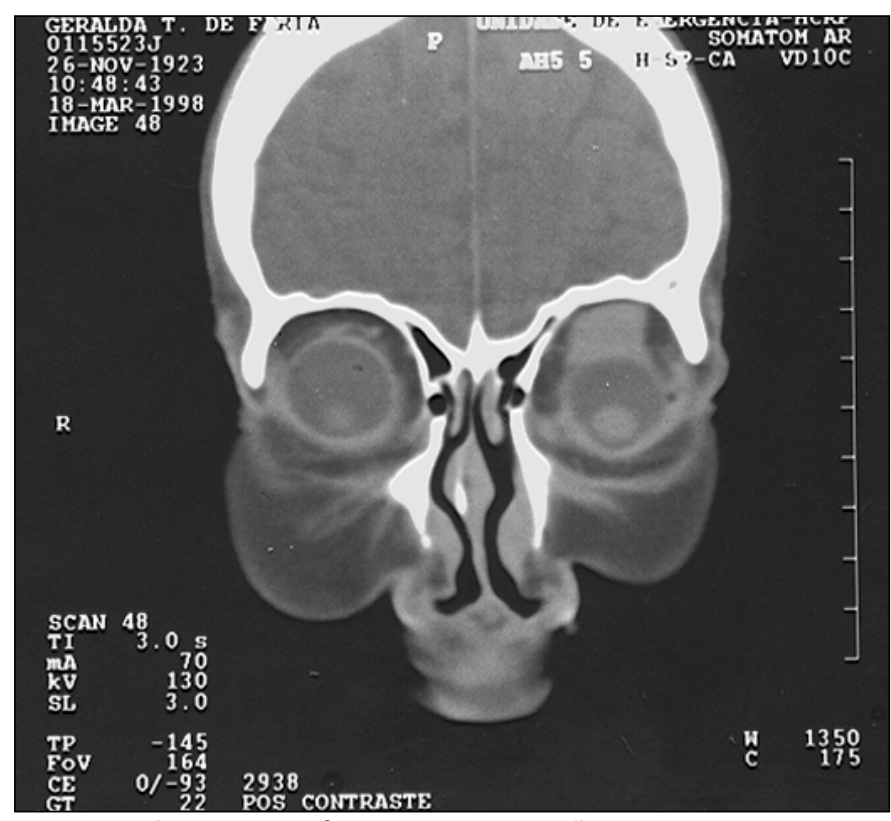

Figura 1 - Corte tomográfico coronal da lesão revelando massa bem delimitada, superior e adjacente ao globo ocular leve grau de pleomorfismo e de atipia celular. Não foram observadas invasão de vasos sangüíneos, necrose ou hemorragia intra-tumoral. Havia cerca de 5 mitoses/10 campos de 400X. Para a análise imunohistoquímica, foram utilizados os marcadores vimentina, antígeno comum leucocitário (LCA), citoceratinas (AE1 e AE3), proteína filamentar S100, fator VIII, actina muscular (HHF35) e reticulina. O material mostrou-se positivo apenas para a vimentina e reticulina, sendo que a reticulina revelou uma trama espessa individualizando as células e espaços vasculares, o que praticamente selou o diagnóstico de hemangiopericitoma (Figura 2).

A paciente vem sendo seguida ambulatorialmente a cada 6 meses, e dois anos após a cirurgia, a tomografia não exibe sinais de recidiva. As pálpebras estão simétricas, os olhos bem posicionados e as versões sem anormalidades. A exoftalmetria é de 7,0 $\mathrm{mm}$ em ambos os olhos.

\section{DISCUSSÃO}

Embora sejam de ocorrência rara, os hemangiopericitomas devem ser lembrados no diagnóstico diferencial das massas orbitárias bem delimitadas, que apresentam, nos estudos tomográficos, reforço à administração de contraste. Estas características também são vistas em outras lesões, como nos hemangiomas cavernosos, schwannomas, neurofibromas e histiocitomas fibrosos. De forma menos comum, os hemangiopericitomas também podem apresentar, à tomografia, calcificações intra-lesionais, erosão óssea e evidência de grandes vasos arteriais nutridores ${ }^{(5)}$.

As angiografias de hemangiopericitomas localizados fora da órbita demonstram que esses tumores são ricamente vascularizados $^{(6-7)}$. Nas ultrasonografias, geralmente eles são vistos como lesões sólidas, bem delimitadas, de baixa a média refletividade, que podem apresentar estrutura interna irregular, às custas de componentes císticos. Quando utiliza-se ressonância magnética, as lesões são melhor visíveis em T1,

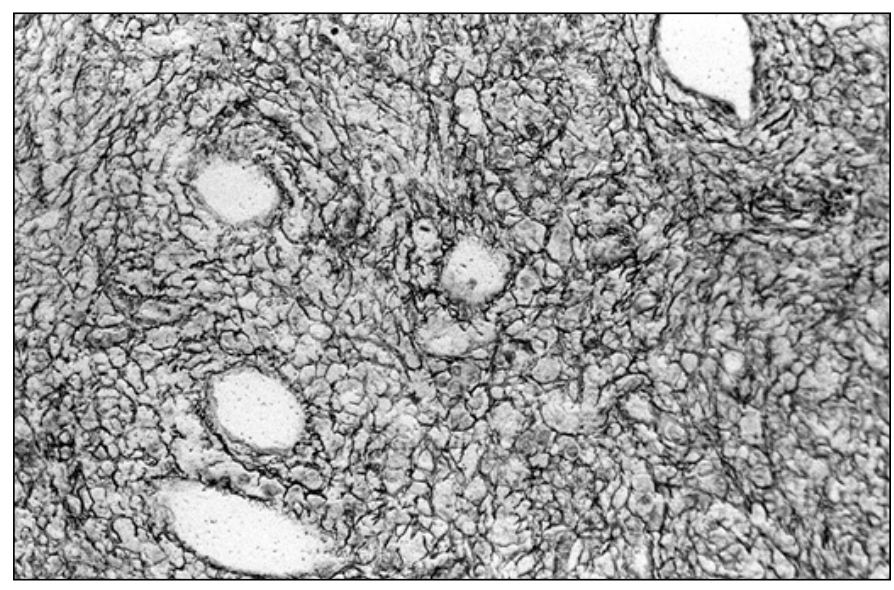

Figura 2 - Corte histopatológico da lesão revelando depósito de reticulina individualizando as células tumorais e os espaços sangüíneos 
como massas hipointensas em relação à gordura orbitária, que podem realçar com o uso do Gadolínio ${ }^{(5)}$.

O quadro clínico dos hemangiopericitomas orbitários é invariante. Em praticamente todos os casos encontra-se proptose de evolução lenta e de longa duração ${ }^{(8)}$. É o mesmo comportamento dos hemangiopericitomas que ocorrem fora da órbita, que costumam apresentar-se como massa indolor ${ }^{(7)}$. Estando na órbita, os hemangiopericitomas causam, pelo efeito compressivo, dor, diplopia e redução da acuidade visual. Outras manifestações típicas de massa intraorbitária podem ainda ser vistas, como dobras de coróide, edema de papila, congestão de vasos retinianos e neuropatia óptica, além de edema e equimose de pálpebras ${ }^{(5)}$.

No caso apresentado, a queixa da paciente era toda dirigida para a presença da massa na pálpebra superior. A ausência de proptose provavelmente deveu-se à localização anterior da lesão na órbita, o que explica também a ocorrência da blefaroptose por compressão do músculo elevador da pálpebra, uma vez que a simples retirada do tumor normalizou o posicionamento palpebral.

De interesse para o oftalmologista, é útil saber que, além da órbita, os hemangiopericitomas também já foram encontrados no saco lacrimal(6), justaposto ao globo ocular ${ }^{(9)}$, na glândula lacrimal ${ }^{(10)}$, nas meninges do nervo óptico ${ }^{(11)}$, e como massa subconjuntival ou subcutânea, no canto interno da fenda palpebral.

Como não apresenta características clínicas patognomônicas, o diagnóstico acaba sendo inteiramente baseado na histopatologia, que pode apresentar algumas dificuldades de caracterização. Com efeito, a lesão pode confundir-se com histiocitoma fibroso, hemangioendotelioma maligno, meningioma angioblástico e condrossarcoma ${ }^{(4,12-13)}$. Isto se deve ao fato de que os pericitos não exibem características marcantes à microscopia óptica. Assim, o diagnóstico é feito pela observação do padrão arquitetural assumido pelas células à microscopia óptica, pelos detalhes celulares à microscopia eletrônica ${ }^{(2)}$, ou pelo uso da imunohistoquímica, havendo quem defenda que o uso da coloração para reticulina seja indispensável ${ }^{(13)}$.

Análises imunohistoquímicas referem que estes tumores também reagem com a vimentina, o antígeno CD34 e a proteína S-100 ${ }^{(5,12,14)}$. À microscopia eletrônica, as células do hemangiopericitoma possuem núcleo grande e citoplasma pobre em organelas. Identificam-se numerosos processos citoplasmáticos alongados. De forma característica, observa-se que cada célula, individualmente, é separada das células adjacentes por uma membrana basal( ${ }^{(7)}$.

Embora descrevam-se critérios de malignidade para estes tumores, observa-se que tumores histologicamente benignos acabam apresentando um comportamento maligno e viceversa $^{(3)}$. A presença de células tumorais gigantes, pleomorfismo celular, focos de hemorragia e necrose, grande número de mitoses por campo (mais do que 35 mitoses/campo em aumento de $400 \mathrm{X}^{(3,8)}$, alto grau de celularidade e invasão vascular têm sido associados a tumores clinicamente agressi- $\operatorname{vos}^{(4,12)}$. De acordo com esses critérios, o caso relatado apresentava quadro histológico benigno.

Alguns estudos relatam a associação entre hemangiopericitomas e hipoglicemia, sendo que esta desaparece após ressecções parciais ou totais de tumor ${ }^{(7)}$. Investigações em Biologia Molecular ${ }^{(15-16)}$ sugerem que este fenômeno possa ser decorrente da produção de fatores de crescimento semelhantes à insulina, que além de causar hipoglicemia, estimulam o crescimento tumoral de uma forma autócrina.

Quanto ao tratamento, é unânime a recomendação de exérese total da lesão, com margens amplas ${ }^{(2,7-8)}$. Há relatos do uso de radioterapia e de quimioterapia para lesões recidivantes, com resultados variáveis ${ }^{(17-19)}$. Infelizmente observase que, mesmo as lesões bem delimitadas, que sofreram completa ressecção, podem evoluir com recidiva local, muitas vezes $\operatorname{tardia}^{(3)}$. Na maior série de casos de hemangiopericitomas orbitais, que contou com 30 pacientes $^{(8)}$, a taxa de recorrência foi de $30.0 \%$. O tempo entre o diagnóstico e a recidiva variou de 1 mês $^{(7)}$ a 18 anos $^{(13)}$. Portanto, é mandatório o seguimento prolongado de todos os pacientes, independente do quadro histológico.

\section{ABSTRACT}

Purpose: To describe a rare case of orbital hemangiopericytoma. Methods: Case report of an association of blepharoptosis with orbital hemangiopericytoma. Results: When the lesion was surgically removed the position of the upper eyelid returned to normal. Conclusions: Anterior orbital lesions can lead to blepharoptosis by compression of the levator palpebrae muscle.

Keywords: Hemangiopericytoma; Orbital neoplasms/etiology; Blepharoptosis/complications

\section{REFERÊNCIAS}

1. Stout AP, Murray MR. Hemangiopericytoma. A vascular tumor featuring Zimmerman's pericytes. Ann Surg 1942:26-33.

2. Shields JA Vasculogenic tumors and malformations. In: Shields JA, Diagnosis and management of orbital tumors. Philadelphia: WB Saunders; 1989. p. 132-4.

3. Jacobiec FA, Bilyk JR, Font RL. Vascular tumors and malformations. In: Spencer WH, Ophthalmic pathology - an atlas and textbook. Philadelphia: WB Saunders; 1996. p. 2545-9.

4. Rodgers IR, Grove Jr AS. Vascular lesions of the orbit. In: Albert DM, Jacobiec FA, Principles and Practice of Ophthalmology: Clinical Practice. Philadelphia: WB Saunders, 1994. p. 1971-2.

5. Karcioglu ZA, Nasr AM, Haik BG. Orbital hemangiopericytoma: clinical and morphologic features. Am J Ophthalmol 1997;124:661-72.

6. Roth SI, August CZ, Lissner GS, O'Grady RB. Hemangiopericytoma of the lacrimal sac. Ophthalmology 1991;98:925-7.

7. Enzinger FM, Smith BH. Hemangiopericytoma: an analysis of 106 cases. Hum Pathol 1976;7:61-82.

8. Croxatto JO, Font RL. Hemangiopericytoma of the orbit: a clinicopathologic study of 30 cases. Hum Pathol 1982;13:210-8.

9. Lee JT, Pettit TH, Glasgow BJ. Epibulbar hemangiopericytoma. Am J Ophthalmol 1997;124:547-9. 
10. Redmond RM, Mannor GE, Garner A, Rose GE. Lacrimal gland hemangiopericytoma. Am J Ophthalmol 1995;119:99-100.

11. Boniuk M, Messmer EP, Font K. Hemangiopericytoma of the meninges of the optic nerve: a clinicopathologic report including electron microscopic observations. Ophthalmology 1985;92:1780-7.

12. Middleton LP, Duray PH, Merino MJ. The histological spectrum of hemangiopericytoma: application of immunohistochemical analysis including proliferating markers to facilitate diagnosis and predict prognosis. Hum Pathol 1998;29:636-40.

13. Jakobiec FA, Howard GM, Jones IR, Wolff M. Hemangiopericytoma of the orbit. Am J Ophthalmol 1974;78:816-34.

14. Khouqeer ZA, Figueiredo AP, Correia CP, Oliver KM, Burnier Jr MN. Immunohistochemical profile of hemangiopericytomas of the orbit. IOVS 2000;41:S125.

15. Paveli K, Paveli ZP, Cabdjan T, Karner I, Samarzija M, Stambrook PJ.
Insulin-like growth factor family in malignant haemangiopericytomas: the expression and role of insulin-like growth factor I receptor. J Pathol 1999; 188:69-75.

16. Pavelic K, Spaventi S, Gluncic V, Matejcic A, Pavicic D, Karapandza N, Kusic Z, Lukac J, Dohoczky C, Cabrijan T, Pavelic J. The expression and role of insulin-like growth factor II in malignant hemangiopericytomas. J Mol Med 1999;77:865-9.

17. Setzkorn RK, Lee DJ, Iliff NT, Green R. Hemangiopericytoma of the orbit treated with conservative surgery and radiotherapy. Arch Ophthalmol 1987; 105:1103-5.

18. Wong PP, Yagoda A Chemotherapy of malignant hemangiopericytoma. Cancer 1978;41:1256-60.

19. Mira JG, Chu FC, Fortner JG. The role of radiotherapy in the management of malignant hemangiopericytoma: report of eleven new cases and review of the literature. Cancer 1977;39:1254-9.

\title{
I CONGRESSO BRASILEIRO DE CATARATA E CIRURGIA REFRATIVA
}

\author{
21 a 23 de Junho de 2.001
}

\section{CENTRO DE CULTURA E CONVENÇÕES DE GOIÂNIA}

REALIZAÇÃO: Sociedade Brasileira de Catarata e Implantes Intraoculares Sociedade Brasileira de Cirurgia Refrativa Sociedade Brasileira de Administração em Oftalmologia

SECRETARIA EXECUTIVA: Promove Eventos Especiais

Fone: (0xx62) 215-8200 - Fax: (0xx62) 215-8063

e-mail: promove@terra.com.br 